\title{
BIBLIOGRAPHIE PARTIELLE DES OEUVRES
}

\section{DE PHILOSOPHIE EN LANGUE FRANÇAISE}

\section{POUR L'ANNEE 1994}

Abbé Pierre, Albert Jacquard. Absolu: dialogue. Animé par Hélène Amblard. Seuil, 1994.

L'activité de la pensée: émergences et troubles. D. Anzieu, B. Gibello, D. Houzel et al. Dunod, 1994.

Aimé, Jacqueline. Les moutons d'Abel ou Un monde sans amour. Ed. Osmondes, 1994.

Ainsi parlaient les anciens: in honorem Jean-Paul Dumont. Mélanges réunis par Lucien Jerphagnon et publ. par Jacqueline Lagrée et Daniel Delattre. Presses universitaires de Lille, 1994.

Alberoni, Francesco. L'érotisme. Trad. de l'italien par Raymonde Coudert. Pocket, 1994.

Allard, Robert. La jeunesse de Pascal: de la légende à l'histoire. J. Lagneau, 1994.

Althusser, Louis. Ecrits philosophiques et politiques. Tome I. Textes réunis et prés. par François Matheron. Stock, 1994.

- Sur la philosophie. Gallimard, 1994.

Andrieu, Bernard. Les cultes du corps: éthique et sciences. Ed. de l'Harmattan, 1994.

Angoulvent, Anne-Laure. L'esprit baroque. PUF, 1994.

- Hobbes et la morale politique. PUF, 1994.

Anthropologie de la lumière. Etudes réunies par Danielle Morali. Presses universitaires de Nancy, 1994.

Anzieu, Didier. Le penser: du moi-peau au moi-pensant. Dunod, 1994.

Aristote. De l'âme. Texte établi par A. Jannone; trad. et notes de E. Barbey. Gallimard, 1994.

- Histoire des animaux. Trad., présentation et notes de Janine Betier. Gallimard, 1994.

- Problèmes. Tome III, sections XXVIII à XXXVIII et index. Texte établi et traduit par Pierre Louis. Les Belles lettres, 1994.

. Traité du temps: Physique, livre IV, 10-14. Introd., trad. et commentaire par Catherine Collobert. Ed. Kimé, 1994.

Avantages et désavantages de la découverte de l'Amérique: Chastellix. Raynal et le concours de l'Académie de Lyon. Textes réunis et 
commentés par Hans-Jürgen Lüsebrink et Alexandre Mussard. Publ. de l'Université de Saint-Etienne, 1994.

Babut, Daniel. Parerga: choix d'articles (1974-1994). Lyon: Maison de l'Orient méditerranéen, 1994.

Bailhache, Gérard. Le sujet chez Emmanuel Lévinas: fragilité et subjectivité. PUF, 1994.

Balibar, Etienne. Lieux et noms de la vérité. La Tour-d'Aigues: Ed. de l'Aube, 1994.

Barreau, Jean-Claude. Quelle morale pour aujourd'hui. Plon, 1994.

Baudrillard, Jean. Figures de l'altérité. Descartes et Cie, 1994.

Baudouin, Jean. La philosophie politique de Karl Popper. PUF, 1994.

Bayle, Pierre. Pensées diverses sur la comète. Ed. critique par A. Prat. $2 \mathrm{e}$ éd. revue par P. Rétat. Société des textes français modernes, 1994.

Beaubatie, Yannick. Le nihilisme et la morale de Nietzsche. Larousse, 1994.

Benasayag, Miguel. Penser la liberté: la décision, le hasard et la situation. Ed. la Découverte, 1994.

Benoist, Jean-Marie. Marx est mort. Préf. de Emmanuel Le Roy Ladurie. PUF, 1994.

Benoist, Jocelyn. Autour de Husserl: l'ego et la raison. J. Vrin, 1994.

Béresniak, Daniel. Les bas-fonds de l'imaginaire: fascisme, intégrisme, ésotérisme et manipulation. Detrad, 1994.

Bergeret, Jean. La violence et la vie: la face cachée de l'oedipe. Payot, 1994.

Bernard, Jean. La bioéthique: un exposé pour comprendre, un essai pour réfléchir. Flammarion, 1994.

Bernet, Rudolf. La vie du sujet: recherches sur l'interprétation de Husserl dans la phénoménologie. PUF, 1994.

Bidima, Jean-Godefroy. Théorie critique et modernité négro-africaine: de l'Ecole de Francfort à la Docta spes africana. Publ. de la Sorbonne, 1994.

Binoche, Bertrand. Les trois sources des philosophies de l'histoire: 17641798 . PUF, 1994.

Boillot, Hervé. 50 modèles de dissertations philosophiques. Alleur (Belgique): Marabout, 1994.

Bonnaud, Robert, La morale et la raison: une histoire universelle. Ed. Kimé, 1994.

Bontpart, Robert. L'homme seul au monde. Editic, 1994.

Bost, Hubert. Pierre Bayle et la raligion. PUF, 1994.

Boullant, François. "Le rire". Henri Bergson. Bernard Lacoste, 1994.

Bourdieu, Pierre. Raisons pratiques: sur la théorie de l'action. Seuil, 1994.

Boutang, Pierre. Dialogues: sur le mythe d'Antigone, sur le sacrifice 
d'Abraham. J.-C. Lattès, 1994.

Bouveresse-Quillot, René. Leibniz. PUF, 1994.

Bresse, Pierre. La symphonie éternelle de l'évolution: une théorie sur le temps du changement. ALTESS, 1994.

Brossat, Alain, dir. Michel Foucault, les jeux de la vérité et du pouvoir: études transeuropéennes. Presses universitaires de Nancy, 1994.

Bugault, Guy. L'Inde pense-t-elle? PUF, 1994.

Buzon, Frédéric de et Vincent Carraud. Descartes et les "Principia" II: corps et mouvement. PUF, 1994.

Caïn, Jacques. L'incohérent, l'inachevé, le plaisir. PUF, 1994.

Campion, Pierre. Mallarmé: poésie et philosophie. PUF, 1994.

Canteins, Jean. Dédale et ses oeuvres. Maisonneuve et Larose, 1994.

Casati, Roberto. La philosophie du son. Nìmes: J. Chambon, 1994.

Castello, Martine. Nos pensées créent le monde: comment les sciences de pointe conduisent à une nouvelle métaphysique. R. Laffont, 1994.

Cavaillès, Jean. Oeuvres complètes de philosophie des sciences. Présentation de Bruno Huisman; In memoriam par Georges Canguilhem. Hermann, 1994.

Celier, Grégoire. Le dieu mortel: invitation à la philosophie. Eguelshardt: Ed. Fideliter, 1994.

Chaboudez, Gisèle. Le concept du phallus dans ses articulations lacaniennes. Lysimaque, 1994.

Chabreuil, Fabien. L'ennéagramme: dynamique de connaissance et d'évolution. Filinges: Carthame éd., 1994.

Chalumeau, Jean-Luc. Les théories de l'art: philosophie, critique et histoire de l'art de Platon à nos jours. Vuibert, 1994.

Chanteur, Janine. La paix: un défi contemporain. Ed. l'Harmattan, 1994.

Châtelet, François. Hegel. Seuil, 1994.

Cheng, Heng. Le tao de l'amour: la méthode du dragon rouge. A. Michel, 1994.

Chopelin, Nicole. Hommes et femmes: l'identité relationnelle de l'être humain. Lyon: PROFAC, 1994.

Chaunu, Pierre. L'axe du temps. Julliard, 1994.

Chemama, Roland. Eléments lacaniens pour une psychanalyse au quotidien. Ed. de l'Association freudienne internationale, 1994.

Colette, Jacques. L'existentialisme. "Que sais-je?" PUF, 1994.

- Kierkegaard et la non-philosophie. Gallimard, 1994.

Collet, François. Pascal: des repères pour situer l'auteur et ses écrits. Nathan, 1994.

Coorebyter, Vincent de, dir. Rhètoriques de la science. PUF, 1994.

Cordier, Françoise. Représentation cognitive et langage: une conquête 
progressive. A. Colin, 1994.

Cornaz, Laurent. L'écriture ou le tragique de la transmission: esquisse pour une histoire de la lettre. Ed. de l'Harmattan, 1994.

Conche, Marcel. Pyrrhon ou l'apparence. PUF, 1994.

Couchard, Françoise. Le fantasme de la séduction dans la culture musulmane: mythes et représentations sociales. PUF, 1994.

Courtine-Denamy, Sylvie. Hannah Arendt. Belfond, 1994.

Cyrulnik, Boris. Les nourritures affectives. France loisirs, 1994.

Daleux, André. Teilhard de Chardin: science et foi réconcilièes? Ed. Gabriandre, 1994.

Danton, Jacques. Méthode de travail pour réussir en philosophie. A. Colin, 1994.

Dastur, Françoise. Dire le temps: esquisse d'une chrono-logie phénoménologique. Fougères: Encre marine, 1994.

- La mort: essai sur la finitude. Hatier, 1994.

Le dernier Schelling: raison et positivité. Sous la dir. de Jean_François Courtine et Jean-François Marquet. J. Vrin, 1994.

David, Michel. Une psychanalyse amusante: Tintin à la lumière de Lacan. Epi-Desclée De Brouwer: la Méridienne, 1994.

Debray, Régis. Manifestes médiologiques. Gallimard, 1994.

Decharneux, Baudouin. L'ange, le devin et le prophète: chemins de la parole dans l'oeuvre de Philon d'Alexandrie dit le Juif. Ed. de l'Université de Bruxelles, 1994.

Les défís de la complexité: vers un nouveau paradigme de la connaissance? Groupe de réflexions transdisciplinaires; Denys de Béchillon, dir.

Ed. l'Harmattan, 1994.

Demaret, Jacques. Le principe anthropique: l'homme est-il le centre de l'univers? A. Colin, 1994.

Le dépassement de soi dans la pensée philosophique. Actes du colloque des 19 et 20 oct. 1990 pour les soixante-dix ans de Fernand Brunner. Neuchâtel: A la Baconnière, 1994.

Derlich, Didier. Séductions. Librairie générale française, 1994.

Derrida, Jacques. Force de loi: le fondement mystique de l'autorité. Galilée, 1994.

- Politiques de l'amitié; suivi de L'oreille de Heidegger. Galilée, 1994.

Destutt de Tracy, Antoine-Louis-Claude. Traité de la volonté et de ses effets;

De l'amour. Textes réunis et revus par Anne Deneys-Tunney et Henry Deneys. Fayard, 1994.

Disselkamp, Annette. "L'éthique protestante" de Max Weber. PUF, 1994. La dissertation philosophique: la didactique à l'oeuvre. Equipe de recherche 
en didactique de la philosophie coordonnée par Françoise Raffin. Préf. de Dina Dreyfus. Hachette éducation, 1994.

Domenach, Jean-Marie. La responsabilité: essai sur le fondement du civisme. Hatier, 1994.

Dor, Joël. Nouvelle bibliographie des travaux de Jacques Lacan. EPEL, 1994.

Ducamp, Jacques. Histoire de la pensée occidentale à partir des grandes philosophies: Antiquité et Moyen âge. Mame, 1994.

Duché, Didier-Jacques. L'éthique ou philosophie morale. Fayard, 1994.

Dunand, Alain. La terre en partage: l'homme en quête de son humanité. Genève: Slatkine, 1994.

Durand, Gilbert. L'imaginaire: essai sur les sciences et la philosophie de l'image. Hatier, 1994.

Duverney, Claude. Le critère de subsomption: l'application des catégories kantiennes. Genève: Slatkine, 1994.

Ehrard, Jean. L idée de nature en France dans la première moitié du XVIIIe siècle. A. Michel, 1994.

Ehrlich, Marie-France. Mémoire et compréhension du langage. Presses universitaires de Lille, 1994.

Elbaz, Sion. Prélude au compromis: philosopher sans gravité. Kimé, 1994.

Elders, Leo J. La métaphysique de Saint Thomas d'Aquin dans une perspective historique. J. Vrin, 1994. Engel, Pascal. Davidson et la philosophie du langage. PUF, 1994.

Emonet, Pierre-Marie. L'âme humaine expliquée aux simples. Chambray: CLD, 1994.

Engel, Pascal. Introduction à la philosophie de l'esprit. Ed. la Découverte, 1994.

Epictète. Manuel. Trad. du grec par Myrto Gondicas; avec un postf. de Alain Nadaud. Ed. Mille et une nuits, 1994.

Erasme. Eloge de la folie. Trad. du latin et présenté par Claude Barousse. Arles: Actes Sud, 1994.

Eribon, Didier. Michel Foucault et ses contemporains. Fayard, 1994.

Fabre, Michel. Penser la formation. PUF, 1994.

Faure, Bernard. La mort dans les religions d'Asie: un exposé pour comprendre, un essai pour réfléchir. Flammarion, 1994.

Faye, Jean-Pierre. Le piège: la philosophie heideggerrienne et le nationalsocialisme. Balland, 1994.

Ferrieres-Pestureau, Suzanne. La métaphore en psychanalyse. Préf. de Monique Schneider. Ed. l'Harmattan, 1994.

Ferry, Jean-Marc. Philosophie de la communication. I, De l'antinomie de la vérité à la fondation ultime de la raison. Ed. du Cerf, 1994.

. Philosophie de la communication. II, Justice politique et démocratie 
procédurale. Ed. du Cerf, 1994.

Ferry, Luc. Le nouvel ordre écologique: l'arbre. l'animal et l'homme.

Librairie générale française, 1994.

Finkielkraut, Alain. Les deux cultures. Palaiseau: Ecole Polytechnique, Département Humanités et sciences sociales, 1994.

Fisette, Denis. Lecture frégéenne de la phénoménologie. Combas: Ed. de l'Eclat, 1994.

Fontenelle, Bernard Le Bouyer de. Entretiens sur la pluralité des mondes. Préf. de Francois Bott. La Tour-d'Aigues: Ed. de l'Aube, 1994.

Forthomme, Bernard. La charite de l'infinitésimal. Cariscript, 1994.

Franck, Robert, dir. Faut-il chercher aux causes une raison?: l'explication causale dans les sciences humaines. J. Vrin, 1994.

Frank, Manfred, Jean-Paul Larthomas, Alexis Philomenko. Sur la troisième "Critique". Textes réunis et présentés par Dominique Janicaud. Combas: Ed. de l'Eclat, 1994.

Frege, Gottlob. Ecrits logiques et philosophiques. Trad. et introd. de Claude Imbert. Nouv. éd. Seuil, 1994.

Friedman, Yona. L'univers erratique: et si les lois de la nature ne suivaient aucune loi? Préf. de Dominique Lecourt. PUF, 1994.

Fruchon, Pierre. L'herméneutique de Gadamer: platonisme et modernité. Ed. du Cerf, 1994.

Gagnebin, Jeanne-Marie. Histoire et narration chez Walter Benjamin. L'Harmattan, 1994.

Gardies, Jean-Louis. Les fondements sémantiques du discours naturel. J. Vrin, 1994.

Gaudemar, Martine de. Leibniz: de la puissance au sujet. J. Vrin, 1994.

Gautier, Jean-François. L univers existe-t-il?: essai. Arles: Actes Sud, 1994. Gilbert Simondon: une pensée de l'individuation de la technique. Colloque organisé par le Collège intemational de philosophie, avril 1992. A. Michel, 1994.

Girard, René. "Quand ces choses commenceront." Entretiens avec Michel Treguer. Arléa, 1994.

Giroud, Françoise et Bernard-Henry Lévy. Les hommes et les femmes. Librairie générale française, 1994.

Glucksmann, André. La fêlure dumonde: éthique et sida. Flammarion, 1994.

Goddard, Jean-Christophe et Bernard Mabille, dir. Le pouvoir. IntégraleVrin, 1994.

Goth, Christian. Initiation aux sciences humaines: philosophie, psychologie, psychanalyse, sociologie, ethnologie. Europsychologies, 1994.

Gourinat, Michel. De la philosophie: classes terminales, classes préparatoires aux grandes écoles, premier cycle d'enseignement 
supérieur. Tomes I et II. Hachette, 1994.

Les grands entretiens du "Monde". Tome II. Penser la philosophie, les sciences, les religions. Avec Paul Ricoeur, Edgar Morin, JeanToussaint Desanti, et al. Préf. de Thomas Ferenczi. "Le Monde" éd., 1994.

Grimaldi, Nicolas. L'ardent sanglot: cinq études sur l'art. Fougères: Encre marine, 1994.

Gros, Frédéric. Autrui: textes expliqués, sujets analysés, glossaire. Hatier, 1994.

Goyard-Fabre, Simone. La construction de la paix ou Le travail de Sisyphe. J. Vrin, 1994.

- Puffendorf et le droit naturel. PUF, 1994.

Goyet, Francis. Rhétorique de la tribu, rhétorique de l'Etat. PUF, 1994.

Gradus philosophique: un répertoired 'introductions méthodiques à la lecture des ouvres. Par Bernard Baertschi, Jean-François Balaudé, Renaud Barbaras, et al. Dir. de Laurent Jaffro et Monique Labrune. Flammarion, 1994.

Guého, Fabrice, Paul Loubière, Fabrice Noval. La philosophie au bac L. A. Michel, 1994.

- La philosophie au bac $S$ et ES. A. Michel, 1994.

Guénon, René. La crise du monde moderne. Gallimard, 1994.

Haar, Michel. L'oeuvre d'art: essai sur l'ontologie des oeuvres. Hatier, 1994.

Halbwachs, Maurice. Les cadres sociaux de la mémoire. Postf. de Gérard Namer. A. Michel, 1994.

Holbach, Paul Henri Dietrich, baron d'. Système social. Texte rev. par Josiane Boulad-Ayoub. Fayard, 1994.

Huber, Gérard. L illusion métabiologique. PUF, 1994.

Huisman, Bruno. Les philosophes et le pouvoir. Dunod, 1994.

Huisman, Denis. La philosophie. Avec André Vergez. Alleur (Belgique): Marabout, 1994.

Philosophie: aide-mémoire: terminales L-ES-S. Nathan, 1994.

Husserl, Edmund. Méditations cartésiennes; et Les conférences de Paris. Présentation, trad. et notes par Marc de Launay. PUF, 1994.

L'idée d'humanité: données et débats. Actes du XXXIVe colloque des intellectuels juifs de langue française. Textes réunis parJean Halpérin et Georges Lévitte. A. Michel, 1994.

L'imaginaire religieux gréco-romain. Ouvrage collectif de l'EPRIL, Equipe pour la recherche sur l'imaginaire de la latinité. Presses universitaires de Perpignan, 1994.

Israël, Lucien. Le désir à l'oeil. Arcanes, 1994. 
Jambou, Philippe. La profondeur de l'ineffable: essai philosophique. Barré et Dayez, 1994.

Jagot, Paul-Clément. Le pouvoir de la volonté: sur soi-même, les autres et le destin. Nouv. éd. ref. et corr. Saint-Jean-de-Braye:Dangles, 1984.

Jankélévitch, Vladimir. Premières et dernières pages. Avant-propos et bibliogr. de Françoise Schwab. Seuil, 1994.

Jaurès, Jean. De la réalité du monde sensible. Ed. Alcuin, 1994.

Jean, Georges. Voyages en utopie. Avec la collaboration de Nathalie Harrison. Gallimard, 1994.

Jeanneret, Annie. De la science à la vie. Bordeaux: le Castor astral, 1994.

Jeannière, Abel. Platon. Seuil, 1994.

Jodorowsky, Alexandro. La sagesse des blagues. Textes réunis par Layla Bess. Chêne-Bourg (Suisse), Annemasse: Ed. Vivez soleil, 1994.

Jolibert, Bernard. Platon: l'ascèse éducative et l'intérêt de l'âme. Ed. l'Harmattan, 1994.

Jolivet, Jean, ed. Abélard ou La philosophie du langage. Fribourg: Ed. universitaires, 1994.

Jouary, Jean-Paul. Entrer en philo. Stock, 1994.

Kant, Immanuel. La religion dans les limites de la simple raison. Trad. J. Gibelin; nouv. éd. rev., introd., annotée et indexée par M. Naar. J. Vrin, 1994.

- Remarques touchant les observations sur le sentiment du beau et du sublime. Trad., introd. et annotées par Brigitte Geonget; préf. de Bernard Bourgeois. J. Vrin, 1994.

- Théorie et pratique; D'un prétendu droit de mentir par l'humanité; La fin de toutes choses; et autres textes. Introd., trad., notes, bibliogr. et chronologie par Françoise Proust. Flammarion, 1994.

Karsenti, Bruno. Marcel Mauss: le fait social total. PUF, 1994.

Kekenbosch, Christiane. La mémoire et le langage. Nathan, 1994.

Kerisel, Françoise. Le soleil de Diogène: les penseurs de la Grèce antique. Hatier, 1994.

Kofman, Sarah. L'énigme de la femme: la femme dans les textes de Freud. Librairie générale française, 1994.

- Le mépris des juifs: Nietzsche, les juifs, l'antisémitisme. Galilée, 1994.

Kremer-Marietti, Angèle. La philosophie cognitive. "Que sais-je?" PUF, 1994.

Kunzmann, Peter. Atias de la philosophie. Trad. de Zoé Housez et Stéphane Robillard. France loisirs, 1994.

Lacan, Jacques. La relation d'objet. Texte établi par Jacques-Alain Miller. Seuil, 1994.

Lacarrière, Jacques. Les gnostiques. A. Michel, 1994. 
Lacoste, Jean-Yves. Expérienceet absolu: questions disputées sur l'humanité de l'homme. PUF, 1994.

Lacroix, Jean-Yves. L'utopie: philosophie de la nouvelle Terre. Présentation de Jacques Moutaux. Bordas, 1994.

Laforgue, René. Au delà du scientisme. G. Trédaniel, 1994.

Lallement, Daniel-Joseph. L'échec du marxisme sonne-t-il le glas de l'humanisme? P. Téqui, 1994.

Langhade, Jacques. Du Coran à la philosophie: la langue arabe et la formation du vocabulaire philosophique de Farabi. Préf. de Jean Jolivet. Damas: Institut français de Damas, 1994.

Larroque, Michel. Volonté et involonté dans la pensée occidentale et orientale. Ed. l'Harmattan, 1994.

Laupies, Frédéric. Premières leçons sur le pouvoir. PUF, 1994.

Laurent, Alain. L'individualisme méthodologique. PUF, 1994.

Lecercle, Jean-Jacques. Frankenstein, mythe et philosophie. 2e éd. corr. PUF, 1994.

Lecouteux, Claude. Mondes parallèles: l'univers des croyances du Moyen âge. H. Champion, 1994.

Lécuru, Denis. Thésaurus Lacan. Vol. I. Citations d'auteurs dans l'ensemble de l'oeuvre écrite. EPEL, 1994.

Le Gaufey, Guy. L'éviction de l'origine. EPEL, 1994.

Lefebvre, Guy. Les inextricables tribulations de la fonction concevante. $\mathrm{La}$ pensée universelle, 1994.

Lefranc, Jean. La philosophie: textes expliqués, sujets analysés, glossaire. Hatier, 1994.

Legendre, Pierre. Dieu au miroir: étude sur l'institution des images. Fayard, 1994.

Leibniz, Gottfried Wilhelm. Système nouveau de la nature et de la communication des substances et autres textes. Présentation et notes de Christiane Frémont. Flammarion, 1994.

Le Moigne, Jean-Louis. Le constructivisme. Tome I. Des fondements. ESF éd., 1994.

Leroux, Pierre. Aux philosophes, aux artistes, aux politiques: trois discours et autres textes. Texte établi et préf. par Jean-Pierre Lacassagne. Payot, 1994.

Le Ru, Véronique. D'Alembert philosophe. J. Vrin, 1994.

Lesourd, Serge. Adolescences, rencontre du féminin: essai psychanalytique sur la différence des sexes. Ramonville-Saint-Ange: Erès, 1994.

Lévinas, Emmanuel. Les imprévus de l'histoire. Fontfroide-le-Haut: Fata Morgana, 1994.

- Liberté et commandement. Fata Morgana, 1994. 
Lévy, Pierre. L'intelligence collective: pour une anthropologie du cyberspace. Ed. la Découverte, 1994.

Leyenberger, Georges. La philosophie de Hölderlin. Ed. Osiris, 1994.

Libera, Alain de. La philosophie médiévale. 3ème éd. PUF, 1994.

Lieux et non-lieux de l'imaginaire. Arles: Actes Sud, 1994.

Linssen, Robert. L'univers: corps d'un seul vivant. ALTESS, 1994.

Lombard, Jean. Aristote, politique et éducation. Ed. l'Harmattan, 1994.

Lorient, Hugues. La logique de l'interprétation. Le Cercle du phénix bleu, 1994.

Lutz-Fuchs, Dominique. Psychothérapies de femmes africaines: Mali. Ed. l'Harmattan, 1994.

Lyotard, Jean-François. Dérive à partir de Marx et Freud. Galilée, 1994. - Des dispositifs pulsionnels. Galilée, 1994.

Macherey, Pierre. Introduction à l'"Ethique" de Spinoza: la cinquième partie, les voies de la libération. PUF, 1994.

Maler, Henri. Congédier l'utopie?: l'utopie selon Karl Marx. L'Harmattan, 1994.

Maritain, Jacques. Oeuvres complètes. Vol. XIV (1921-1944). Jacques et Raïssa Maritain, eds. Fribourg (Suisse): Ed. universitaires, 1994.

Marx, Karl. Philosophie. Ed établie et annotée par Maverick, Ken. La fracture de l'être. Les Deux océans, 1994.

Maximilien Rubel. Gallimard, 1994.

Mengue, Philippe. Gilles Deleuze ou Le système du multiple. Ed. Kimé, 1994.

Meyer, Michel, dir. La philosophie angl-saxonne. PUF, 1994.

Michel, Alain. La parole et la beauté: rhétorique et esthétique dans la tradition occidentale. A. Michel, 1994.

Michon, Cyrille. Nominalisme: la théorie de la signification d'Occam. J. Vrin, 1994.

Misrahi, Robert. La problématique du sujet aujourd'hui. La Versanne: Encre marine, 1994.

Mongin, Olivier. Face au scepticisme: 1976-1993: les mutations du paysage intellectuel ou l'invention de l'intellectuel démocratique. Ed. la Découverte, 1994.

. Paul Ricoeur. Seuil, 1994.

Montaigne, Michel de. Les essais. Textes choisis, présentés, annotés et commentés par Denis A. Canal. Larousse, 1994.

Moreau, Pierre-François. Spinoza: l'expérience et l'éternité. PUF, 1994.

Morfaux, Louis-Marie. Philosophie: terminale scientifique: notions et textes. A. Colin, 1994.

Morin, Edgar. La complexité humaine. Textes rassemblés et présentés par Heinz Weinmann. Flammarion, 1994. 
Mourral, Isabelle. Précis de philosophie pour le monde technique. Ed. universitaires, 1994.

Mouton, Joseph. Sois artiste: traité de politique esthétique. Aubier, 1994.

Muglioni, Jean-Michel. Réponse à la question, "Qu'est-ce que les Lumieres?" Hatier, 1994.

M'Uzan, Michel de. La bouche de l'inconscient: essais sur l'interprétation. Gallimard, 1994.

Nabert, Jean. L'expérience intérieure de la liberté: et autres essais de philosophie morale. Préf. de Paul Ricoeur. PUF, 1994.

Nakos, Jean. L'itinéraire d'un profane: essai. Lyon: C. de Ramaix, 1994.

Nancy, Jean-Luc. Les muses. Galilée, 1994.

Narbonne, Jean-Marc. La métaphysique de Plotin. J. Vrin, 1994.

Nasio, Juan David. Cing leçons sur la théorie de Jacques Lacan. Payot, 1994.

Navet, Georges. Pierre Leroux, politique, socialisme et philosophie. Publ. de la Sociéte P.-J. Proudhon, 1994.

Nédélec, Françoise. Le sida au quotidien: de l'épreuve aux stratégies de vie. Ed. I'Harmattan, 1994.

Negri, Antonio. Spinoza subversif: variations (in)actuelles. Kimé, 1994.

Nietzsche, Friedrich. L'antéchrist. Trad. inédite, introd., notes, bibliogr. et index par Eric Blondel. Flammarion, 1994.

- Introduction aux leçons sur l'"Oedipe-roi" de Sophocle: été 1870; Introduction aux études de philologie classique: été 1871. Trad. par Françoise Dastur et Michel Haar; prés. par Michel Haar. La Versanne: Encre marine, 1994.

- La naissance de la tragédie. Trad. nouvelle et présentation de Cornélius Heim. Denoël, 1994.

- La naissance de la tragédie: ou hellénisme et pessimisme. Trad. de Jean Marnold et Jacques Morland; introd. et notes d'Angèle KremerMarietti. Librairie générale française, 1994.

Nolane, Richard D. La chair et le sang: vampires et vampirisme. UGE, 1994. Ollender, Maurice. Les langues du Paradis: Aryens et Sémites, un couple providentiel. Seuil, 1994.

Où est le bonheur? Cinquième Forum "Le Monde" Le Mans. Textes réunis et prés. par Roger-Pol Droit. "Le Monde" éd., 1994.

Où va la modernité? Groupe de recherche pour l'éducation et la prospective. Toulouse: Ed. GREP Midi-Pyrénées, 1994

Parker, Steve. Aristote et la pensée scientifique. Ed. du Sorbier, 1994.

Pascal, Blaise. Discours sur les passions de l'amour. Postf. de Jérôme Vérain. Ed. Mille et une nuits, 1994.

Paul, Jean-Marie. Dieu est mort en Allemagne: des Lumières à Nietzsche. 
Payot, 1994.

Peillon, Vincent. La tradition de l'esprit: itinéraire de Maurice Merieau-

Ponty. B. Grasset, 1994.

Pena-Ruiz, Henri. Philosophie: la dissertation. Nouv. éd. Bordas, 1994.

Perron, Roger. Le complexe d'Oedipe. PUF, 1994.

Philibert, Myriam. Stonehenge et son secret. Le Grand livre du mois, 1994.

Philippe, Marie-Dominique. Philosophie de l'art. Tome II. Ed. universitaires, 1994.

Philonenko, Alexis. Bergson ou De la philosophie comme science rigoureuse. Ed. du Cerf, 1994.

- Relire Descartes. J. Grancher, 1994.

Philosopher sans frontières: hommage à Joachim Kopper. Centre Gaston Bachelard et la société bourguignonne de philosophie. Ed. universitaires de Dijon, 1994.

Philosophie contemporaine en France. Ministère des affaires étrangères, Direction générale des relations culturelles, scientifiques et techniques. Avant-propos, Yves Mabin; réd. par Christian Descamps, Jocelyn Benoist, Eric Alliez. ADPF, 1994.

Platon. Phédon. Préf. et comment. par Agnès Forestier-Nordmann. Pocket, 1994.

Théétète. Trad. inédite, introd. et notes par Michel Narcy. Flammarion, 1994.

Plotin. Traité 9. Introd., trad., comment. et notes par Pierre Hadot. Ed. du Cerf, 1994.

Le pouvoir. Sous la dir. de Geneviève Dewulf; préf. de Régis Debray.

Presses universitaires de Nancy, 1994.

Le pouvoir. Sous la coord. de Gilbert Guislain. Rosny: Bréal, 1994.

Pratique de la philosophie de $A$ à $Z$. Elisabeth Clément, Chantal Demonque, Laurent Hansen-Love, Pierre Kahn. Hatier, 1994.

Poliakov, Léon. Le mythe aryen: essai sur les sources du racisme et des nationalismes. Pocket, 1994.

Pottier, Richard. Essai d'anthropologie du mythe. Ed. Kimé, 1994.

Pozin, Amaud. Etre humain: une introduction à la roue de connaissance. Les Deux océans, 1994.

Proust, Françoise. L'histoire à contretemps: le temps historique chez Walter Benjamin. Ed. du Cerf, 1994.

Puig, Jaume de. Les sources de la pensée philosophique de Raimond Sebond. H. Champion, 1994.

La puissance et son ombre: de Pierre Lombard à Luther. Textes trad. et commentés par Olivier Boulnois, Jean-François Genest, Elizabeth Karger. Aubier, 1994. 
Quillien, Jean, éd. La réception de la philosophie allemande en France aux XIXe et XXe siècles. Presses universitaires de Lille, 1994.

Rabineau, Isabelle. Modernes libertins: un art de la résistance. Bordeaux: le Castor astral, 1994.

Raikovic, Pierre. Le sommeil dogmatique de Freud: Kant. Schopenhauer. Freud. Le Plessis-Robinson: Synthélabo, 1994.

Rekel, Jean-François. Histoire de la philosophie occiddentale: de Thales à Kant. Nil éd., 1994.

Rey, Louis, dir. Ethique et spiritualité de l'environnement: séminaire international, Rabat, Maroc, 28-30 avril 1992. G. Trédaniel, 1994.

Richard Rorty: ambiguités et limites du postmodernisme. Dir. scientifique et présent. par Gilbert Hottois et Maurice Weyembergh. J. Vrin, 1994.

Ricoeur, Paul. Aux frontières de la philosophie. Seuil, 1994.

Roche, Christian. Philosophie, terminale S. Bordas, 1994. Philosophie: L, ES. Nouv. éd. Bordas, 1994.

Rochlitz, Rainer. Subversion et subvention: art contemporain et argument esthétique. Gallimard, 1994.

Rosenberg, Patrice. Philosophie: terminales STT. STI, STL, SMS. Nathan, 1994.

Rozenberg, Jacques J. Philosophie et folie: fondements psychanalytiques de la métaphysique. Ed. l'Harmattan, 1994.

Roudinesco, Elisabeth. Généalogies. Fayard, 1994.

Russ, Jacqueline. Abrégé de philosophie: terminales littéraire, économique et sociale. A. Colin, 1994.

- Abrégé de philosophie: terminales STT-SMS-STL-STI. A. Colin, 1994.

- La pensée éthique contemporaine. PUF, 1994.

. Les théories du pouvoir. Librairie générale française, 1994.

Said, Suzanne. Approches de la mythologie grecque. Nathan univ., 1994.

Samuel-Scheyder, Monique, dir. Image de l'homme: l'Allemagne au XVle siècle. Actes du colloque du Centre de recherches germaniques et scandinaves de l'Univ. de Nancy 2, mars 1991. Presses universitaires de Nancy, 1994.

Schlanger, Jacques. Gestes de philosophes. Aubier, 1994.

Schwaller de Lubicz, René Adolphe. Du symbole et de la symbolique. Dervy, 1994.

Sède, Gérard de. L'occultisme dans la politique: de Pythagore à nos jours. R. Laffont, 1994.

Sénèque. Traités. Présentation et notes de Guy Rachet; trad. d'Elias Regnault. France loisirs, 1994.

Serres, Michel. Atlas. Julliard, 1994. 
Serrurier, Catherine. Que sont nos maris devenus?: la nouvelle guerre entre les sexes. Desclée De Brouwer, 1994.

Sève, Bernard. La question philosophique de l'existence de Dieu. PUF, 1994.

Sève, Lucien. Pour une critique de la raison bioèthique. O. Jacob, 1994.

Seymour, Michel. Pensée, langage et communauté: une perspective antiindividualiste. Montréal: Bellarmin, Paris: Vrin, 1994.

Simon, Michel. La peau de l'âme: intelligence artificielle, neurosciences. Ed. du Cerf, 1994.

Sipos, Joël. Lacan et Descartes: la tentation de la métaphysique. PUF, 1994.

Sorel, Reynal. Les cosmogonies grecques. PUF, 1994.

Souche-Dagues, Denise. Recherches hégeliennes: infini et dialectique. J. Vrin, 1994.

Spinoza, Baruch. Traité des authorités théologique et politique. Trad. et annoté par Madeleine Francès. Gallimard, 1994.

Stanguennec, André. Raison analytique et raison dialectique dans la pensée post-kantienne. L'Age d'homme, 1994.

Stenger, Gerhardt. Nature et liberté chez Diderot: après l'"Encyclopédie". Universitas, 1994.

Stiegler, Bernard. La faute d'Epiméthée. Galilée, 1994.

La synchronicité, l'âme et la science. H. Reeves, M. Cazenave, P. Solié et al. A. Michel, 1994.

Tape, Goze. L'intelligence en Afrique: une étude du raisonnement expérimental. L'Harmattan, 1994.

Tenzer, Nicolas. Philosophie politique. PUF, 1994.

Terray, Emmanuel. Une passion allemande: Luther, Kant, Schiller, Holderlin, Kleist. Seuil, 1994.

Terrel, Jean. Hobbes, matérialisme et politique. J. Vrin, 1994.

Testard, Jacques. Le désir du gène. Flammarion, 1994.

Thomas, Tom. Une brève histoire de l'individu. Albatroz, 1994.

Tiry, Gérard. Connaître le réel: mythes ou réalités. Lyon: Chronique sociale, 1994.

Todorov, Tzvetan. Face â l'extrême. Seuil, 1994.

Tosel, André. Du matérialisme de Spinoza. Ed. Kimé, 1994.

Touati, Armand, dir. Violences: penser, agir, transformer. Marseille: "Le journal des psychologues," 1994.

Tozzi, Michel. Penser soi-même: initiation à la philosophie. Bruxelles: Vie ouvrière, 1994.

Trotignon, Pierre. Les philosophes français de 1945 à 1965 . 5e éd. PUF, 1994.

Troude-Chastenet, Patrick, dir. Sur Jacques Ellul: un penseur de notre temps. 
Bordeaux-le-Bouscat: l'Esprit du Temps, 1994.

Utopie et utopies: l'imaginaire du projet social européen. Actes du séminaire européen d'études des idées et de l'imaginaire collectif, Bordeaux, 1989-1990. Textes réunis par Claude-Gilbert Dubois. Mont-deMarsan: Ed. Interuniversitaires, 1993-1994.

Valdman, Edouard. Les Juifs et l'argent: pour une métaphysique de l'argent. Galilée, 1994.

Vauvenargues, Luc de Clapiers. Fragments sur Montaigne. Manuscrit édité et précédé d'une étude "Vauvenargues et le philosophes" par Jean

Dagen. H. Champion, 1994.

Vaysse, Jean-Marie. Totalité et subjectivité: Spinoza dans l'idéalisme allemand. J. Vrin, 1994.

Vieillard-Baron, Jean-Louis, dir. De saint Thomas à Hegel. PUF, 1994. - Qu'est-ce que l'éducation? Montaigne, Fichte et Lavelle. J. Vrin, 1994.

Vignaux, Georges. Les sciences cognitives: une introduction. Librairie générale française, 1994.

Violences, racines ou destins des pulsions? Sous la dir. de Joyce Aïn. Ramonville: Erès, 1994.

Virilio, Paul. Esthétique de la disparition. Librairie gén. française, 1994.

Voltaire. Dictionnaire philosophique. Ed. présentée et annotée par Alain Pons. Gallimard, 1994.

- Dictionnaire philosophique. Présentation, notes et annexes par Béatrice Didier. Impr. nationale, 1994.

Wahl, Jean. Du rôle de l'idée de l'instant dans la philosophie de Descartes. Descartes \& Cie, 1994.

Warin, François. Nietzsche et Bataille: la parodie à l'infini. PUF, 1994.

Xénophon. Banquet; suivi de Apologie de Socrate. Textes établis et trad. par François Ollier. Gallimard, 1994.

Yakira, Elhanan. La causalité: de Galilée à Kant. PUF, 1994.

Zarader, Jean-Pierre. Petite histoire des idées philosophiques; Le statut de l'oeuvre d'art chez André Malraux. Ellipses, 1994.

Zima, Peter Vaclav. La déconstruction: une critique. PUF, 1994. 